\title{
Retardation effects from quark confinement on low-energy nucleon dynamics
}

\author{
Renat Kh.Gainutdinov and Aigul A.Mutygullina \\ Department of Physics \\ Kazan State University, \\ 18 Kremlevskaya St, Kazan 420008, \\ Russia \\ E-mail: Renat.Gainutdinov@ksu.ru
}

\section{Introduction}

Study of the effects of quarks and gluons confined within hadrons on nucleon dynamics is of fundamental importance in understanding the strong interaction. These effects are important for describing the short-range (SR) part of the $N N$ interactions. They can also be very important for describing the dynamics of dense nucleon matter especially at large densities. As has been shown in Ref.[1], the internal quark structure of baryons can have substantial effects on the composition and structure of neutron star matter. Since a nonperturbative treatment of quantum chromodynamics (QCD) is not possible today, the effects of the internal quark structure of hadrons on low-energy nucleon dynamics are investigated by using quark models (see, for instance, Refs.[2-9]). However, these models completely miss the effects of retardation in the gluon-exchange interaction, despite the fact that they can be important [10], and on the whole the effects of quark-gluon retardation on nucleon dynamics are poorly understood at present.

The effects of quark-gluon retardation on nucleon dynamics differ profoundly from the well-known meson-retardation effects that are taken into account, for example, in the Bonn model [11]. Meson retardation effects give rise to nonlocality in time of the $N N$ interaction, and hence to an energy dependence of the effective potentials describing these interactions, which can have significant effects on three- and many nucleon results [12]. Nonlocality in time of such interactions is an expression of a loss of probability from the two-nucleon subspace of the Hilbert space of hadron states. Obviously, quark-gluon retardation, that has to be taken into account in describing hadron dynamics, should also result in nonlocality in time of hadron interactions. However, due to confinement this must not lead to a loss of probability from the Hilbert space of hadron states. On the other hand, as is well known, 
of a quantum system may be nonlocal in time only in the case when the system is not closed, and as a result the evolution is not unitary. Similar problems arising due to nonlocality in time of the $N N$ interaction has been discussed in [13-20]. To solve these problems quantum dynamics need to be extended to describe the unitary evolution of a closed system in the case where the interaction generating the dynamics of the system is nonlocal in time. For the first time, this problem has been solved in Ref.[21], where it has been shown that the use of the Feynman approach $[22,23]$ to quantum theory in combination with the canonical approach allows such an extension of quantum dynamics. The generalized quantum dynamics (GQD) developed in this way has been shown $[24,25]$ to open new possibilities for describing hadron dynamics.

In the present paper the GQD is used for investigating quark-gluon retardation effects on low-energy hadron dynamics. We show that retardation from quark confinement results in an anomalous off-shell behavior of the two-nucleon amplitudes and in a lack of continuity of the evolution operator describing low-energy hadron dynamics that for this reason is not governed by the Schrödinger equation. In Sec.II we discuss some problems in describing hadron dynamics that arise due to quark-gluon retardation effects. The principal features of the GQD are reviewed in Sec.III, and in Sec.IV we show that the GQD provides an extension of Hamiltonian dynamics which can describe the evolution of quantum systems with confined degrees of freedom. The existence of such degrees of freedom gives rise to a peculiar dynamical situation that allows one to conclude that retardation effects of quark confinement on low-energy hadron can be significant. In Sec.IV this fact is illustrated by the example of the dynamics of nucleons with internal structure described by a constituent quark model. In Sec.VI we use the GQD to construct a model that is a generalization of the separable-potential model to the case when the $N N$ interaction is nonlocal in both space and time. We show that despite its simplicity the model, in which retardation effects of quark confinement are taken into account, yields the nucleon-nucleon phase shifts in good agreement with experiment. Analyzing the off-shell behavior of the two-nucleon amplitudes in the case where the $N N$ interaction is nonlocal in time, we find that quark-gluon retardation can have significant effects on three- and many-nucleon results. 


\section{Retardation effects and low-energy hadron dynamics}

According to QCD, the physics of the strong interaction exhibits different relevant excitations at distinct length (or momentum) scales. At short space-time distances $(r<<1 \mathrm{fm})$ the relevant degrees of freedom are quarks and gluons, effectively unconfined due to asymptotic freedom. At large distances $(r>>1 \mathrm{fm})$, on the other hand, hadronic degrees of freedom should be considered as relevant degrees of freedom. At the same time, due to confinement there are no observables, which can be associated with the quark and gluon degrees of freedom at least in the low-energy regime. However, in principle this does not mean that the quark and gluon degrees of freedom are not relevant in this regime. The above means only that there are no observables associated with quarks and gluons in the low-energy regime. To clarify this point, note the following. The most fundamental aspect of quantum theory in its present interpretation is its probabilistic character. The description of a physical system requires two kinds of elements, the observables and the states of the system. The states in turn are described by the vectors of a Hilbert space. The state vector determines the probabilities of finding some values of the observables, when a measurement is performed, and can be expanded in terms of the eigenvectors corresponding to the eigenvalues of a complete system of commuting observables. From this and the phenomenon of quark confinement, according to which quarks and gluons are not observable in free states, it follows that vectors describing low-energy states of a system of strongly interacting particles can be expanded in terms of eigenvectors associated with hadronic degrees of freedom, i.e. they belong to the Hilbert space of hadron states. The time evolution of the system is described by the evolution equation

$$
\left|\psi(t)>=U\left(t, t_{0}\right)\right| \psi\left(t_{0}\right)>
$$

where $\mid \psi(t)>$ is a state vector of the Hilbert space of hadron states, and $U\left(t, t_{0}\right)$ is the evolution operator defined on this space. Since due to confinement quark-gluon retardation effects must not lead to a loss of probability from the Hilbert space, $U\left(t, t_{0}\right)$ must be a unitary operator

$$
U^{+}\left(t, t_{0}\right) U\left(t, t_{0}\right)=U\left(t, t_{0}\right) U^{+}\left(t, t_{0}\right)=\mathbf{1} .
$$

Here we use the interaction picture. Thus due to confinement the time evolution of a system of strongly interacting particles at low energies can be described by the unitary evolution operator defined on the Hilbert space of hadron states. Nevertheless, the quark and gluon degrees of freedom may be relevant in describing low-energy hadron dynamics, since they should manifest 
Let us now show that quarks and gluons confined within hadrons can have substantial effects on low-energy hadron dynamics. One of the fundamental requirement of quantum theory is that the evolution operator must satisfy the composition law

$$
U\left(t, t^{\prime}\right) U\left(t^{\prime}, t_{0}\right)=U\left(t, t_{0}\right), \quad U\left(t_{0}, t_{0}\right)=\mathbf{1}
$$

In the case of an isolated system, the evolution operator in the Schrödinger picture $U_{s}\left(t_{2}, t_{1}\right) \equiv \exp \left(-i H_{0} t_{2}\right) U\left(t_{2}, t_{1}\right) \exp \left(i H_{0} t_{1}\right)$ depends on the difference $\left(t_{2}-t_{1}\right)$ only, so that the operators $V(t) \equiv U_{s}(t, 0)$ constitute a one-parameter group of unitary operators, with the group property

$$
V\left(t_{1}+t_{2}\right)=V\left(t_{1}\right) V\left(t_{2}\right), \quad V(0)=\mathbf{1} .
$$

Here and below, we use the units in which $c=\hbar=1$, and $H_{0}$ is the free Hamiltonian. If the evolution operator is assumed to be strongly continuous, i.e. if

$$
\lim _{t_{2} \rightarrow t_{1}}\left\|V\left(t_{2}\right)\left|\psi>-V\left(t_{1}\right)\right| \psi>\right\|=0
$$

then from Stone's theorem it follows that this one-parameter group has a self-adjoint infinitesimal generator $H$ :

$$
V(t)=\exp (-i H t), \quad i d / d t V(t)=H V(t) .
$$

Identifying $H$ with the total Hamiltonian as usual, we get the time-dependent Schrödinger equation: $i \frac{d \mid \psi_{s}(t)>}{d t}=H \mid \psi_{s}(t)>$, where $\left|\psi_{s}(t)>=V(t)\right| \psi_{s}(t=0)>$ is a state vector in the Schrödinger picture. Thus, in this case, the interaction being described by the interaction Hamiltonian is necessarily instantaneous. Obviously, the infinitesimal generator $H$ being the operator of the total energy cannot contain terms depending on energy i.e. on its spectral parameter. From this it follows that, if the continuity condition (5) is satisfied, then the time evolution of a system can be unitary only in the case when the interaction generating the dynamics of a quantum system is instantaneous. Thus the evolution operator describing low energy hadron dynamics cannot be strongly continuous, since due to quark-gluon retardation effects hadron interactions should be nonlocal in time, and, on the other hand, due to confinement the evolution operator must be unitary. This means that quark-gluon retardation and confinement must result in the fact that the evolution of hadron systems cannot be governed by the Schrödinger equation.

We have shown that quark-gluon retardation effects give rise to the fact that the evolution operator describing hadron dynamics cannot be strongly 
requirement that the evolution operator must be strongly continuous is used as a fundamental assumption (due to Stone's theorem it is equivalent to the assumption that the evolution of a quantum system is governed by the Schrödinger equation), this requirement is not necessary on physical grounds, and it is enough to require that

$$
<\psi_{2}\left|V\left(t_{2}\right)\right| \psi_{1}>\underset{t_{2} \rightarrow t_{1}}{\rightarrow}<\psi_{2}\left|V\left(t_{1}\right)\right| \psi_{1}>
$$

for any physically realizable states $\left|\psi_{1}\right\rangle$ and $\left|\psi_{2}\right\rangle[26]$. Note, in this connection, that there are normalized vectors in the Hilbert space that represent the states for which the energy of a system is infinite. Such states cannot be considered as physically realizable, and hence the corresponding matrix elements of the evolution operator need not be continuous. From this it follows that in principle Hamiltonian dynamics can be extended to the case where only the physical continuity condition (6) is satisfied. As has been shown in Ref.[21], this really can be done by using the Feynman approach to quantum theory in combination with the canonical approach. As we show bellow, the GQD developed in this way allows one to take into account quark-gluon retardation in describing hadron dynamics.

\section{Generalized Quantum Dynamics}

In the GQD the following assumptions are used as basic postulates:

(i) The physical state of a system is represented by a vector (properly by a ray) of a Hilbert space.

(ii) An observable $\mathrm{A}$ is represented by a Hermitian hypermaximal operator $\alpha$. The eigenvalues $a_{r}$ of $\alpha$ give the possible values of A. An eigenvector $\mid \varphi_{r}^{(s)}>$ corresponding to the eigenvalue $a_{r}$ represents a state in which A has the value $a_{r}$. If the system is in the state $|\psi\rangle$, the probability $P_{r}$ of finding the value $a_{r}$ for $\mathrm{A}$, when a measurement is performed, is given by

$$
P_{r}=<\psi\left|P_{V_{r}}\right| \psi>=\sum_{s}\left|<\varphi_{r}^{(s)}\right| \psi>\left.\right|^{2},
$$

where $P_{V_{r}}$ is the projection operator on the eigenmanifold $V_{r}$ corresponding to $a_{r}$, and the sum $\Sigma_{s}$ is taken over a complete orthonormal set $\mid \varphi_{r}^{(s)}>(\mathrm{s}=1,2, \ldots)$ of $V_{r}$. The state of the system immediately after the observation is described by the vector $P_{V_{r}} \mid \psi>$.

These assumptions are the main assumptions on which quantum theory is founded. In the canonical formalism these postulates are used together with the assumption that the time evolution of a state vector is governed 
used. Instead the assumptions (i) and (ii) are used together with the following postulate.

(iii) The probability of an event is the absolute square of a complex number called the probability amplitude. The joint probability amplitude of a time-ordered sequence of events is product of the separate probability amplitudes of each of these events. The probability amplitude of an event which can happen in several different ways is a sum of the probability amplitudes for each of these ways.

The statements of the assumption (iii) express the well known law for the quantum-mechanical probabilities. Within the canonical formalism this law is derived as one of the consequences of the theory. However, in the Feynman formulation of quantum theory this law is directly derived starting from the analysis of the phenomenon of quantum interference, and is used as a basic postulate of the theory. According to the assumption (iii), the probability amplitude of an event which can happen in several different ways is a sum of contributions from each alternative way. In particular, the amplitude $<\psi_{2}\left|U\left(t, t_{0}\right)\right| \psi_{1}>$ can be represented as a sum of contributions from all alternative ways of realization of the corresponding evolution process. Dividing these alternatives in different classes, we can then analyze such a probability amplitude in different ways. For example, subprocesses with definite instants of the beginning and end of the interaction in the system can be considered as such alternatives. In this way the amplitude $<\psi_{2}\left|U\left(t, t_{0}\right)\right| \psi_{1}>$ can be written in the form [21]

$$
<\psi_{2}\left|U\left(t, t_{0}\right)\right| \psi_{1}>=<\psi_{2}\left|\psi_{1}>+\int_{t_{0}}^{t} d t_{2} \int_{t_{0}}^{t_{2}} d t_{1}<\psi_{2}\right| \tilde{S}\left(t_{2}, t_{1}\right) \mid \psi_{1}>,
$$

where $<\psi_{2}\left|\tilde{S}\left(t_{2}, t_{1}\right)\right| \psi_{1}>$ is the probability amplitude that if at time $t_{1}$ the system was in the state $\left|\psi_{1}\right\rangle$, then the interaction in the system will begin at time $t_{1}$ and will end at time $t_{2}$, and at this time the system will be in the state $\mid \psi_{2}>$. More precisely, $<\psi_{2}\left|\tilde{S}\left(t_{2}, t_{1}\right)\right| \psi_{1}>$ is the density of the probability amplitude of the above event, and as contributions to $<\psi_{2}\left|U\left(t, t_{0}\right)\right| \psi_{1}>$ one has to consider amplitudes $<\psi_{2}\left|\tilde{U}_{\varepsilon}\left(t_{2}, t_{1}\right)\right| \psi_{1}>=\int_{t_{2}}^{t_{2}+\varepsilon} d t_{2}^{\prime} \int_{t_{1}}^{t_{1}+\varepsilon} d t_{1}^{\prime} \theta\left(t_{2}^{\prime}-t_{1}^{\prime}\right)<\psi_{2}\left|\tilde{S}\left(t_{2}^{\prime}, t_{1}^{\prime}\right)\right| \psi_{1}>$, where $\varepsilon<<t_{2}-t_{1}$ and

$$
\theta(\tau)=\left\{\begin{array}{c}
1, \text { for } \quad \tau>0 \\
0, \text { for } \quad \tau<0
\end{array}\right.
$$

The amplitude $<\psi_{2}\left|\tilde{U}_{\varepsilon}\left(t_{2}, t_{1}\right)\right| \psi_{1}>$ is the amplitude that if at time $t_{1}$ the system was in the state $\left|\psi_{1}\right\rangle$, then the interaction in the system will begin in time internal $t_{1}, t_{1}+\varepsilon$ and will end in time interval $t_{2}, t_{2}+\varepsilon$, and at the 
an operator-valued generalized function of $t_{1}$ and $t_{2}$ [21]. Nevertheless, it is convenient to call $\tilde{S}\left(t_{2}, t_{1}\right)$ an "operator", using this word in generalized sense. In the case of an isolated system the operator $\tilde{S}\left(t_{2}, t_{1}\right)$ can be represented in the form $[21]$

$$
\tilde{S}\left(t_{2}, t_{1}\right)=\exp \left(i H_{0} t_{2}\right) \tilde{T}\left(t_{2}-t_{1}\right) \exp \left(-i H_{0} t_{1}\right) .
$$

To clarify the role, which the operator $\tilde{S}\left(t_{2}, t_{1}\right)$ plays in the GQD, note the following. The Feynman formulation is based on the assumption that the history of a system can be represented by some path in space-time. From the postulate (iii) it then follows that the probability amplitudes of any event is a sum of the probability amplitudes that a particle has a completely specified path in space-time. The contribution from a single path is postulated to be an exponential whose (imaginary) phase is the classical action (in units of $\hbar$ ) for the path in question. In the GQD the history of a system is represented by the version of the time evolution of the system associated with completely specified instants of the beginning and end of the interaction in the system. Such a description of the history of a system is more general and requires no supplementary postulates like the above assumptions of the Feynman formulation. On the other hand, the probability amplitudes $<\psi_{2}\left|\tilde{S}\left(t_{2}, t_{1}\right)\right| \psi_{1}>$, in terms of which we describe quantum dynamics, are used in the spirit of Feynman's theory: The probability amplitude of any event is represented as a sum of these amplitudes.

As has been shown in Ref.[21], for the evolution operator $U\left(t, t_{0}\right)$ given by (7) to be unitary for any times $t_{0}$ and $t$, the operator $\tilde{S}\left(t_{2}, t_{1}\right)$ must satisfy the following equation:

$$
\left(t_{2}-t_{1}\right) \tilde{S}\left(t_{2}, t_{1}\right)=\int_{t_{1}}^{t_{2}} d t_{4} \int_{t_{1}}^{t_{4}} d t_{3}\left(t_{4}-t_{3}\right) \tilde{S}\left(t_{2}, t_{4}\right) \tilde{S}\left(t_{3}, t_{1}\right)
$$

Note that, since $\tilde{S}\left(t_{2}, t_{1}\right)$ may be only an operator-valued distribution, in general the production $\tilde{S}\left(t_{2}, t_{4}\right) \tilde{S}\left(t_{3}, t_{1}\right)$ is not defined at $t_{4}=t_{3}$. However, this does not lead to any problems because in Eq.(9) the above production is multiplied by the factor $t_{4}-t_{3}$. Eq.(9) allows one to obtain the operators $\tilde{S}\left(t_{2}, t_{1}\right)$ for any $t_{1}$ and $t_{2}$, if the operators $\tilde{S}\left(t_{2}^{\prime}, t_{1}^{\prime}\right)$ corresponding to infinitesimal duration times $\tau=t_{2}^{\prime}-t_{1}^{\prime}$ of interaction are known. Thus this equation allows one to obtain the contributions to the evolution operator from the processes associated with any duration times of interaction if those from the processes associated with infinitesimal duration times of interactions are known. It is natural to assume that most of the contribution to the evolution operator in the limit $t_{2} \rightarrow t_{1}$ comes from the processes associated with an 
by $H_{\text {int }}\left(t_{2}, t_{1}\right)$, the operator $\tilde{S}\left(t_{2}, t_{1}\right)$ can be represented in the form

$$
\tilde{S}\left(t_{2}, t_{1}\right)=H_{\text {int }}\left(t_{2}, t_{1}\right)+\tilde{S}_{1}\left(t_{2}, t_{1}\right),
$$

where $\tilde{S}_{1}\left(t_{2}, t_{1}\right)$ is the part of the operator $\tilde{S}\left(t_{2}, t_{1}\right)$ which in the limit $t_{2} \rightarrow t_{1}$ gives a negligibly small contribution to the evolution operator in comparison with $H_{\text {int }}\left(t_{2}, t_{1}\right)$. Within the GQD the operator $H_{\text {int }}\left(t_{2}, t_{1}\right)$ plays the role which the interaction Hamiltonian plays in the ordinary formulation of quantum theory: It generates dynamics of a system. This operator can be regarded as a generalization of the interaction Hamiltonian, and it was called the generalized interaction operator. Obviously, the operator $H_{\text {int }}\left(t_{2}, t_{1}\right)$ must satisfy Eq.(9) in the limit $t_{2} \rightarrow t_{1}$

$$
F\left(t_{2}, t_{1}\right) \underset{t_{2} \rightarrow t_{1}}{\rightarrow} 0
$$

where

$$
F\left(t_{2}, t_{1}\right)=-\left(t_{2}-t_{1}\right) H_{\text {int }}\left(t_{2}, t_{1}\right)+\int_{t_{1}}^{t_{2}} d t_{4} \int_{t_{1}}^{t_{4}} d t_{3}\left(t_{4}-t_{3}\right) H_{\text {int }}\left(t_{2}, t_{4}\right) H_{\text {int }}\left(t_{3}, t_{1}\right) .
$$

If $H_{\text {int }}\left(t_{2}, t_{1}\right)$ is specified, Eq.(9) allows one to find the operator $\tilde{S}\left(t_{2}, t_{1}\right)$. Formula (7) can then be used to construct the evolution operator $U\left(t, t_{0}\right)$ and accordingly the state vector

$$
|\psi(t)>=| \psi\left(t_{0}\right)>+\int_{t_{0}}^{t} d t_{2} \int_{t_{0}}^{t_{2}} d t_{1} \tilde{S}\left(t_{2}, t_{1}\right) \mid \psi\left(t_{0}\right)>
$$

at any time $t$. The corresponding evolution operator is of the form [21]

$$
U\left(t, t_{0}\right)=\mathbf{1}+\frac{1}{2 \pi} \int_{-\infty}^{\infty} d x \frac{\exp \left[-i\left(z-H_{0}\right) t\right] T(z) \exp \left[i\left(z-H_{0}\right) t_{0}\right]}{\left(z-H_{0}\right)\left(z-H_{0}\right)},
$$

where $z=x+i y, x$ and $y$ are real, and $y>0$, and the operator $T(z)$ is defined by

$$
T(z)=i \int_{0}^{\infty} d \tau \exp (i z \tau) \tilde{T}(\tau)
$$

with $\tilde{T}(\tau)=\exp \left(-i H_{0} t_{2}\right) \tilde{S}\left(t_{2}, t_{1}\right) \exp \left(i H_{0} t_{1}\right)$. Thus Eq.(9) can be regarded as an equation of motion for states of a quantum system. It should be noted that in the GQD the continuity condition (6) is not used as a basic postulate, and plays only the role of a consistency condition of the theory: Matrix elements of the evolution operator (7) obtained by solving Eq.(9) must be continuous for any physically realizable states. It is reasonable to consider such states as the states for which $\left\|H_{0} \mid \psi>\right\|<\infty$. Thus the condition (6) must be satisfied for all vector $\mid \psi>\in \mathcal{D}\left(H_{0}\right), \mathcal{D}\left(H_{0}\right)$ being domain of $H_{0}$.

From the mathematical point of view the requirement that $H_{\text {int }}\left(t_{2}, t_{1}\right)$ 
means that the operator $H_{\text {int }}\left(t_{2}, t_{1}\right)$ must have such a form that Eq.(9) has a unique solution having the following behavior near the point $t_{2}=t_{1}$ :

$$
\tilde{S}\left(t_{2}, t_{1}\right) \underset{t_{2} \rightarrow t_{1}}{\rightarrow} H_{\text {int }}\left(t_{2}, t_{1}\right)+o\left(\tau^{\epsilon}\right)
$$

where $\tau=t_{2}-t_{1}$ and the value of $\epsilon$ depends on the form of the operator $H_{\text {int }}\left(t_{2}, t_{1}\right)$. Since $\tilde{S}\left(t_{2}, t_{1}\right)$ and $H_{\text {int }}\left(t_{2}, t_{1}\right)$ are only operator-valued distributions, the mathematical meaning of the condition (15) needs to be clarified. We will assume that the condition (15) means that

$$
\begin{gathered}
<\psi_{2}\left|\left(\int_{t_{0}}^{t} d t_{2} \int_{t_{0}}^{t_{2}} d t_{1} \tilde{S}\left(t_{2}, t_{1}\right)\right)\right| \psi_{1}>\underset{t \rightarrow t_{0}}{\rightarrow} \\
<\psi_{2}\left|\left(\int_{t_{0}}^{t} d t_{2} \int_{t_{0}}^{t_{2}} d t_{1} H_{\text {int }}\left(t_{2}, t_{1}\right)\right)\right| \psi_{1}>+o\left(\tau^{\alpha+2}\right), \quad \tau=t-t_{0},
\end{gathered}
$$

for any vectors $\mid \psi_{1}>$ and $\mid \psi_{2}>$ of the Hilbert space. Note that the condition (11) has to be considered in the same sense.

To clarify the above statement, note that Eq.(9) is equivalent to the following equation [21]:

$$
\frac{d T(z)}{d z}=T(z) G^{(2)}(z) T(z)
$$

with

$$
G^{(2)}(z)=-\sum_{n} \frac{|n><n|}{\left(z-E_{n}\right)^{2}}
$$

Here $n$ stands for the entire set of discrete and continuous variables that characterize the system in full, and $|n\rangle$ are the eigenvectors of the free Hamiltonian: $H_{0}\left|n>=E_{n}\right| n>$. Thus, instead of solving Eq.(9), one can solve Eq.(17) for the operator $T(z)$. The operator $\tilde{T}(\tau)$ and correspondingly the operator $\tilde{S}\left(t_{2}, t_{1}\right)$ can then be obtained by using the Fourier transform

$$
<n_{2}|\tilde{T}(\tau)| n_{1}>=-\frac{i}{2 \pi} \int_{-\infty}^{\infty} d x \exp (-i z \tau)<n_{2}|T(z)| n_{1}>
$$

where $z=x+i y, x$ and $y$ are real, and $y>0$. At the same time, the operator $T(z)$ can be directly used for constructing the evolution operator. According to (14) and (15), the operator $T(z)$ has the following asymptotic behavior for $|z| \rightarrow \infty$ :

$$
<n_{2}|T(z)| n_{1}>\underset{|z| \rightarrow \infty}{\rightarrow}<n_{2}|B(z)| n_{1}>+o\left(|z|^{-\beta}\right),
$$

where

$$
B(z)=i \int^{\infty} d \tau \exp (i z \tau) H_{\text {int }}^{(s)}(\tau)
$$


$\beta=1+\epsilon$, and $H_{\text {int }}^{(s)}\left(t_{2}-t_{1}\right)=\exp \left(-i H_{0} t_{2}\right) H_{\text {int }}\left(t_{2}, t_{1}\right) \exp \left(i H_{0} t_{1}\right)$ is the generalized interaction operator in the Schrödinger picture. From (17) and (19) it follows that the operator $B(z)$ must satisfy the following asymptotic condition:

$$
\frac{d<n_{2}|B(z)| n_{1}>}{d z} \underset{|z| \rightarrow \infty}{\rightarrow}<n_{2}\left|B(z) G^{(2)}(z) B(z)\right| n_{1}>+o\left(|z|^{-\beta}\right) .
$$

The above requirements, which the generalized interaction operator has to meet, mean that $B(z)$ must be so close to the solution of equation (17) in the limit $|z| \rightarrow \infty$ that this differential equation has a unique solution having the asymptotic behavior (19). The operator $B(z)$ represents the contribution which $H_{\text {int }}^{(s)}(\tau)$ gives to the operator $T(z)$, and was called the effective interaction operator. It should be also noted, that the operator $T(z)$ satisfies the equation

$$
T\left(z_{1}\right)-T\left(z_{2}\right)=\left(z_{2}-z_{1}\right) \sum_{n} \frac{T\left(z_{2}\right)|n><n| T\left(z_{1}\right)}{\left(z_{2}-E_{n}\right)\left(z_{1}-E_{n}\right)}
$$

provided that $\tilde{S}\left(t_{2}, t_{1}\right)$ satisfies Eq.(9).

As has been shown in Ref.[21], the dynamics governed by Eq.(9) is equivalent to Hamiltonian dynamics in the case where the generalized interaction operator is of the form

$$
H_{\text {int }}\left(t_{2}, t_{1}\right)=-2 i \delta\left(t_{2}-t_{1}\right) H_{I}\left(t_{1}\right),
$$

$H_{I}\left(t_{1}\right)$ being the interaction Hamiltonian in the interaction picture. In this case the state vector $\mid \psi(t)>$ given by (12) satisfies the Schrödinger equation

$$
\frac{d \mid \psi(t)>}{d t}=-i H_{I}(t) \mid \psi(t)>\text {. }
$$

The delta function $\delta(\tau)$ in (21) emphasizes that in this case the fundamental interaction is instantaneous. Thus the Schrödinger equation results from the generalized equation of motion (9) in the case where the interaction generating the dynamics of a quantum system is instantaneous. At the same time, Eq.(9) permits the generalization to the case where the operator $H_{\text {int }}\left(t_{2}, t_{1}\right)$ has no such a singularity as the delta function at the point $t_{2}=t_{1}$ [21]. In this case the fundamental interaction generating the dynamics of a quantum system is nonlocal in time: The evolution operator is defined by $H_{\text {int }}\left(t_{2}, t_{1}\right)$ as a function of the time duration $\tau=t_{2}-t_{1}$ of the interaction. In a more general case, the generalized interaction operator has the following form [25]: 
where the first term on the right-hand side of (22) describes the instantaneous part of the interaction generating the dynamics of a quantum system, while the term $H_{\text {non }}$ represents its nonlocal-in-time part. In order that the dynamics governed by Eq.(9) be different from the dynamics governed by the Schrödinger equation with the interaction Hamiltonian $H_{I}(t)$, the nonlocal operator $H_{n o n}$ must satisfy the condition

$$
\left(t_{2}-t_{1}\right) H_{\text {non }}\left(t_{2}, t_{1}\right) \sim \int_{t_{1}}^{t_{2}} d t_{4} \int_{t_{1}}^{t_{4}} d t_{3}\left(t_{4}-t_{3}\right) H_{\text {non }}\left(t_{2}, t_{4}\right) H_{\text {non }}\left(t_{3}, t_{1}\right), \quad t_{2} \rightarrow t_{1} .
$$

In fact, in the local case, where $H_{\text {int }}\left(t_{2}, t_{1}\right)$ is of the form $(21)$, the first term in the operator $F\left(t_{2}, t_{1}\right)$ is zero, and from the condition (11) it follows that the second term in this operator must tend to zero in the limit $t_{2} \rightarrow t_{1}$ so rapidly as it is required for Eq.(9) to have a unique solution. In the nonlocal case, both terms in the operator $F\left(t_{2}, t_{1}\right)$ are not zero, and, as we show in Sec.IV, they do not decrease when $t_{2} \rightarrow t_{1}$ so rapidly as it is needed. Only their sum can vanish fast enough for the condition (11) to be satisfied. From this it follows that the nonlocal part of the generalized interaction operator must satisfy the condition (23).

The principal feature of the GQD is that it provides an extension of Hamiltonian dynamics which can describe the unitary evolution of a quantum system with confined degrees of freedom. In the next section we will show that the existence of such degrees of freedom, that can manifest themselves only in some retardation of the interaction in the system, gives rise to a peculiar dynamical situation that allows one to conclude that retardation effects of quark confinement on low-energy hadron dynamics can be very significant.

\section{IV.Effects of confined degrees of freedom on the dynamics of a quantum system}

Let us consider the evolution problem for two nonrelativistic particles in the c.m.s. We denote the relative momentum by $\mathbf{p}$ and the reduced mass by $\mu$. Assume that the generalized interaction operator in the Schrödinger picture $H_{\text {int }}^{(s)}(\tau)$ has the form

$$
<\mathbf{p}_{2}\left|H_{\text {int }}^{(s)}(\tau)\right| \mathbf{p}_{1}>=\varphi^{*}\left(\mathbf{p}_{2}\right) \varphi\left(\mathbf{p}_{1}\right) f(\tau),
$$

where $f(\tau)$ is some function of $\tau$, and the form factor $\varphi(\mathbf{p})$ has the following asymptotic behavior for $|\mathbf{p}| \rightarrow \infty$ : 
Let, for example, $\varphi(\mathbf{p})$ be of the form

$$
\varphi(\mathbf{p})=\frac{c_{1}}{|\mathbf{p}|^{\alpha}}+g(\mathbf{p}),
$$

and in the limit $|\mathbf{p}| \rightarrow \infty$ the function $g(\mathbf{p})$ satisfies the estimate $g(\mathbf{p})=$ $o\left(|\mathbf{p}|^{-\delta}\right)$, where $\delta>\alpha, \delta>\frac{3}{2}$. In this case, the problem can be easily solved by using Eq.(15). Representing $<\mathbf{p}_{2}|T(z)| \mathbf{p}_{1}>$ in the form $<\mathbf{p}_{2}|T(z)| \mathbf{p}_{1}>=\varphi\left(\mathbf{p}_{2}\right) \varphi^{*}\left(\mathbf{p}_{1}\right) t(z)$, from (15), we get the equation

$$
\frac{d t(z)}{d z}=-t^{2}(z) \int d^{3} k \frac{|\varphi(\mathbf{k})|^{2}}{\left(z-E_{k}\right)^{2}}
$$

with the asymptotic condition

$$
t(z) \underset{|z| \rightarrow-\infty}{\rightarrow} f_{1}(z)+o\left(|z|^{-\beta}\right),
$$

$f_{1}(z)=i \int_{0}^{\infty} d \tau \exp (i z \tau) f(\tau)$, and $E_{k}=\frac{k^{2}}{2 \mu}$. The solution of Eq.(27) with the initial condition $t(a)=g_{a}$, where $a \in(-\infty, 0)$, is

$$
t(z)=g_{a}\left(1+(z-a) g_{a} \int d^{3} k \frac{|\varphi(\mathbf{k})|^{2}}{\left(z-E_{k}\right)\left(a-E_{k}\right)}\right)^{-1} .
$$

In the case $\alpha>\frac{1}{2}$, the function $t(z)$ tends to a constant as $z \rightarrow-\infty$ :

$$
t(z) \underset{z \rightarrow-\infty}{\rightarrow} \lambda \text {. }
$$

Thus in this case the function $f_{1}(z)$ must tend to $\lambda$ as $z \rightarrow-\infty$. From this it follows that the only possible form of the function $f(\tau)$ is

$$
f(\tau)=-2 i \lambda \delta(\tau)+f^{\prime}(\tau),
$$

where the function $f^{\prime}(\tau)$ has no such a singularity at the point $\tau=0$ as the delta function. In this case the generalized interaction operator $H_{\text {int }}^{(s)}(\tau)$ has the form (21) and hence the dynamics generated by this operator is equivalent to the dynamics governed by the Schrödinger equation with the separable potential

$$
<\mathbf{p}_{2}\left|H_{I}\right| \mathbf{p}_{1}>=\lambda \varphi^{*}\left(\mathbf{p}_{2}\right) \varphi\left(\mathbf{p}_{1}\right) .
$$

Solving Eq.(27) with the boundary condition (30), we easily get the well-known expression for the T matrix in the separable-potential model

$$
<\mathbf{p}_{2}|T(z)| \mathbf{p}_{1}>=\lambda \varphi^{*}\left(\mathbf{p}_{2}\right) \varphi\left(\mathbf{p}_{1}\right)\left(1-\lambda \int d^{3} k \underline{|\varphi(\mathbf{k})|^{2}}\right)^{-1}
$$


Ordinary quantum mechanics does not permit the extension of the above model to the case $\alpha \leq \frac{1}{2}$. Indeed, in the case of such a large-momentum behavior of the form factors $\varphi(\mathbf{p})$, the use of the interaction Hamiltonian given by (31) leads to the ultraviolet divergences, i.e. the integral in (32) is not convergent. We will now show that our formalism allows one to extend this model to the case $0<\alpha<\frac{1}{2}$. Let us determine the class of the functions $f_{1}(z)$ and correspondingly the value of $\beta$ for which Eq.(27) has a unique solution having the asymptotic behavior (28). In the case $\alpha<\frac{1}{2}$, the function $t(z)$ given by (29) has the following behavior for $z \rightarrow-\infty$ :

$$
t(z) \underset{z \rightarrow-\infty}{\rightarrow} b_{1}(-z)^{\alpha-\frac{1}{2}}+b_{2}(-z)^{2 \alpha-1}+o\left(|z|^{2 \alpha-1}\right),
$$

where $b_{1}=-\frac{1}{2} \cos (\alpha \pi) \pi^{-2}\left|c_{1}\right|^{-2}(2 \mu)^{\alpha-\frac{3}{2}}$ and $b_{2}=b_{1}|a|^{\frac{1}{2}-\alpha}-b_{1}^{2}\left(M(a)+g_{a}^{-1}\right)$ with

$$
M(a)=\int \frac{|\varphi(\mathbf{k})|^{2}-\frac{\left|c_{1}\right|^{2}}{|\mathbf{k}|^{2 \alpha}}}{a-E_{k}} d^{3} k .
$$

The parameter $b_{1}$ does not depend on $g_{a}$. This means that all solutions of Eq.(27) have the same leading term in (33), and only the second term distinguishes the different solutions of this equation. Thus, in order to obtain a unique solution of Eq.(27), we must specify the first two terms in the asymptotic behavior of $t(z)$ for $z \rightarrow-\infty$. From this it follows that the functions $f_{1}(z)$ must be of the form

$$
f_{1}(z)=b_{1}(-z)^{\alpha-\frac{1}{2}}+b_{2}(-z)^{2 \alpha-1}
$$

and $\beta=2 \alpha-1$. Correspondingly the functions $f(\tau)$ must be of the form

$$
f(\tau)=a_{1} \tau^{-\alpha-\frac{1}{2}}+a_{2} \tau^{-2 \alpha},
$$

with $a_{1}=-i b_{1} \Gamma^{-1}(1-2 \alpha) \exp \left[i\left(-\frac{\alpha}{2}+\frac{1}{4}\right) \pi\right]$, and $a_{2}=-b_{2} \Gamma^{-1}(1-2 \alpha) \exp (-i \alpha \pi)$, where $\Gamma(z)$ is the gamma-function. This means that in the case $\alpha<\frac{1}{2}$ the generalized interaction operator must be of the form

$$
\begin{gathered}
<\mathbf{p}_{2}\left|H_{\text {int }}^{(s)}(\tau)\right| \mathbf{p}_{1}>=f(\tau) \varphi^{*}\left(\mathbf{p}_{2}\right) \varphi\left(\mathbf{p}_{1}\right)= \\
=a_{1} \varphi^{*}\left(\mathbf{p}_{2}\right) \varphi^{*}\left(\mathbf{p}_{1}\right) \tau^{-\alpha-\frac{1}{2}}+a_{2} \varphi^{*}\left(\mathbf{p}_{2}\right) \varphi\left(\mathbf{p}_{1}\right) \tau^{-2 \alpha},
\end{gathered}
$$

and, as it follows from (29) and (33), for the T matrix we have

$$
<\mathbf{p}_{2}|T(z)| \mathbf{p}_{1}>=N(z) \varphi^{*}\left(\mathbf{p}_{2}\right) \varphi\left(\mathbf{p}_{1}\right)
$$

with 
where $g_{a}=b_{1}^{2}\left(b_{1}|a|^{\frac{1}{2}-\alpha}+a_{2} \Gamma(1-2 \alpha) \exp (i \alpha \pi)-b_{1}^{2} M(a)\right)^{-1}$. It can be easily checked that $N(z)$ can be represented in the following form

$$
N(z)=\frac{b_{1}^{2}}{-b_{2}+b_{1}(-z)^{\frac{1}{2}-\alpha}+M(z) b_{1}^{2}} .
$$

By using (13) and (37), we can construct the evolution operator

$$
\begin{gathered}
<\mathbf{p}_{2}\left|U\left(t, t_{0}\right)\right| \mathbf{p}_{1}>=<\mathbf{p}_{2} \mid \mathbf{p}_{1}>-\frac{i}{2 \pi} \int_{-\infty}^{\infty} d x \\
\times \frac{\exp \left[-i\left(z-E_{p_{2}}\right) t\right] \exp \left[i\left(z-E_{p_{1}}\right) t_{0}\right]}{\left(z-E_{p_{2}}\right)\left(z-E_{p_{1}}\right)} N(z) \varphi^{*}\left(\mathbf{p}_{2}\right) \varphi\left(\mathbf{p}_{1}\right),
\end{gathered}
$$

where $z=x+i y$, and $y>0$. The evolution operator $U\left(t, t_{0}\right)$ defined by (38) is a unitary operator satisfying the composition law (3), provided that the parameter $b_{2}$ is real.

We have stated the correspondence between the form of the generalized interaction operator and the large-momentum behavior of the form factor $\psi(\mathbf{p})$. In the case $\alpha>\frac{1}{2}$, the operator $H_{\text {int }}^{(s)}(\tau)$ would necessarily have the form (21). In this case the fundamental interaction is instantaneous. In the case $0<\alpha<\frac{1}{2}$ (the restriction $\alpha>0$ is necessary for the integral in (29) to be convergent), the only possible form of $H_{\text {int }}^{(s)}(\tau)$ is (36), and hence the interaction generating the dynamics of the system is nonlocal in time. Thus the interaction generating the dynamics can be nonlocal in time only if the form factors have the "bad" large-momentum behavior that within Hamiltonian dynamics gives rise to the ultraviolet divergences.

Let us now show that in the case $\alpha<\frac{1}{2}$ the dynamics generated by the generalized interaction operator (36) is not equivalent to Hamiltonian dynamics. The evolution operator $U\left(t, t_{0}\right)$ given by $(38)$ satisfies the composition law (3) for any $t_{0}, t$ and $t^{\prime}$, provided the operator $\tilde{S}\left(t_{2}, t_{1}\right)$ satisfies Eq.(9) for any $t_{1}$ and $t_{2}$. In the case of model under study the operator $\tilde{S}\left(t_{2}, t_{1}\right)$ is of the form

$$
\tilde{S}\left(t_{2}, t_{1}\right)=D\left(t_{2}, t_{1}\right) \tilde{F}\left(t_{2}, t_{1}\right)
$$

where $\tilde{F}(\tau)$ is a function of $\tau$, and $D\left(t_{2}, t_{1}\right)=\exp \left(i H_{0} t_{2}\right)|\varphi><\varphi| \exp \left(-i H_{0} t_{1}\right)$ is the operator-valued distribution such that

$$
<\mathbf{p}_{\mathbf{2}}\left|D\left(t_{2}, t_{1}\right)\right| \mathbf{p}_{\mathbf{1}}>=\exp \left(i E_{p_{2}} t_{2}\right)<\mathbf{p}_{\mathbf{2}}|\varphi><\varphi| \mathbf{p}_{\mathbf{1}}>\exp \left(-i E_{p_{1}} t_{1}\right),
$$

with $\langle\varphi| \mathbf{p}>=\varphi(\mathbf{p})$. By using (39) and (40), for the function $\tilde{F}(\tau)$ we get the following equation: 


$$
\times<\varphi|\mathbf{k}><\mathbf{k}| \varphi>\tilde{F}\left(t_{2}-t_{4}\right) \tilde{F}\left(t_{3}-t_{1}\right) .
$$

Let us examine this equation in the limit $t_{2} \rightarrow t_{1}$. For this we have to change the variables: $\tau_{i}=\theta_{i} / \nu, \mathbf{k}=\nu \mathbf{q}_{\nu}, i=1,2,3,4$. With such a change of the variables, Eq.(41) can be rewritten in the form

$$
\begin{gathered}
\left(\theta_{2}-\theta_{1}\right) \tilde{F}\left(\theta_{2} / \nu^{2}-\theta_{1} / \nu^{2}\right)=\nu^{-4} \int_{\theta_{1}}^{\theta_{2}} d \theta_{4} \int_{\theta_{1}}^{\theta_{4}} d \theta_{3}\left(\theta_{4}-\theta_{3}\right) \int d^{3} k \exp \left[-i E_{q_{\nu}}\left(\theta_{4}-\theta_{3}\right)\right] \times \\
\times<\varphi\left|\mathbf{q}_{\nu}><\mathbf{q}_{\nu}\right| \varphi>\tilde{F}\left(\theta_{2} / \nu^{2}-\theta_{4} / \nu^{2}\right) \tilde{F}\left(\theta_{3} / \nu^{2}-\theta_{1} / \nu^{2}\right)
\end{gathered}
$$

$\mid \mathbf{q}_{\nu}>$ being the basis vectors such that $<\mathbf{k} \mid \mathbf{q}_{\nu}>=\nu^{-\frac{3}{2}} \delta\left(\mathbf{k}-\nu \mathbf{q}_{\nu}\right)$. The completeness condition for these basis states reads

$$
\int d^{3} q_{\nu}\left|\mathbf{q}_{\nu}><\mathbf{q}_{\nu}\right|=\mathbf{1}
$$

Thus $\mid \mathbf{k}>$ and $\mid \mathbf{q}_{\nu}>$ are eigenstates of the operator $H_{0}$ corresponding to distinct momentum scales. Since $<\left.\varphi\left|\mathbf{q}_{\nu}>=c_{1}\right| \nu \mathbf{q}_{\nu}\right|^{-\alpha}+o\left(\nu^{-\frac{3}{2}-\alpha}\right)$ when $\nu \rightarrow \infty$, by letting $t_{2} \rightarrow t_{1}$, from (42) we get

$$
\begin{gathered}
\left(\theta_{2}-\theta_{1}\right) \tilde{F}\left(\theta_{2} / \nu^{2}-\theta_{1} / \nu^{2}\right)=\nu^{-1-2 \alpha} \int_{\theta_{1}}^{\theta_{2}} d \theta_{4} \int_{\theta_{1}}^{\theta_{4}} d \theta_{3} \tilde{F}\left(\theta_{2} / \nu^{2}-\theta_{4} / \nu^{2}\right) \times \\
\times \tilde{F}\left(\theta_{3} / \nu^{2}-\theta_{1} / \nu^{2}\right) I\left(\theta_{4}-\theta_{3}, \nu\right), \quad \nu \rightarrow \infty
\end{gathered}
$$

with

$$
I(\theta, \nu)=\left|c_{1}\right|^{2} \theta \int d^{3} q_{\nu} \exp \left(-i E_{q_{\nu}} \theta\right)\left|\mathbf{q}_{\nu}\right|^{-2 \alpha}+o\left(\nu^{-\frac{3}{2}+\alpha}\right) .
$$

This expression emphasizes that the relevant momentum scale of intermediate states in Eq.(42) tends to infinity as $t_{2} \rightarrow t_{1}$. In order to demonstrate that Eq.(44) plays the key role in the case $\alpha<\frac{1}{2}$, note that from this equation it follows that in the limit $\tau \rightarrow 0$ the function $\tilde{F}(\tau)$ behaves like $a_{1} \tau^{-\alpha-\frac{1}{2}}$ with

$$
\begin{gathered}
a_{1}=\left(\theta_{2}-\theta_{1}\right)^{\frac{1}{2}-\alpha}\left(\int_{\theta_{1}}^{\theta_{2}} d \theta_{4} \int_{\theta_{1}}^{\theta_{4}} d \theta_{3} I\left(\theta_{4}-\theta_{3}, \infty\right)\right)^{-1}= \\
=\frac{i}{2} \pi^{-2}\left|c_{1}\right|^{-2}(2 \mu)^{\alpha-\frac{3}{2}} \Gamma^{-1}(1-2 \alpha) \cos (\alpha \pi) \exp \left[i\left(-\frac{\alpha}{2}+\frac{1}{4}\right) \pi\right],
\end{gathered}
$$

i.e. this equation determines the main term of the generalized interaction operator (see Eq.(36)). We see from (45) that only the intermediate states with infinite momentum contribute to the parameter $a_{1}$. This manifests itself in the fact that this parameter depends only on the leader term in the form factor (25). Note in this connection that for any vector $|\psi\rangle$ of the Hilbert space $\mathcal{H}$ represented in the form 
with $\psi(\mathbf{k})=<\mathbf{k} \mid \psi>$, one can construct another vector

$$
\left|\psi_{\nu}>=\int \psi(\mathbf{k} / \nu) \nu^{-\frac{3}{2}}\right| \mathbf{k}>d^{3} k,
$$

that represents the same physical state, if we scale $\mathbf{k} \rightarrow \nu \mathbf{k}$, i.e.

$$
\left|\psi_{\nu}>=\int \psi\left(\mathbf{q}_{\nu}\right)\right| \mathbf{q}_{\nu}>d^{3} q_{\nu}
$$

where $\mathbf{q}_{\nu}=\mathbf{k} / \nu$. Varying the parameter $\nu$, we get a set of vectors having the same norm

$$
\left\|\mid \psi_{\nu}>\right\|=\left(\int \psi^{*}\left(\mathbf{q}_{\nu}\right) \psi\left(\mathbf{q}_{\nu}\right) d^{3} q_{\nu}\right)^{\frac{1}{2}}
$$

Each of the vectors $\left|\psi_{\nu}\right\rangle$ belong to the Hilbert space $\mathcal{H}$ even when the parameter $\nu$ is letting to infinity. To clarify this point, let us consider the state $\left|\psi>=\int \psi_{k_{0}}(\mathbf{k})\right| \mathbf{k}>d^{3} k$, where $\psi_{k_{0}}(\mathbf{k})$ is zero everywhere outside the subset $\Delta\left(k_{0}\right)\left(E_{k_{0}} \leq E_{k} \leq E_{k_{0}}+\varepsilon E_{k_{0}}, \varepsilon \ll 1\right)$ of the spectrum of $H_{0}$. Such a state is eigenstate of the projection operator $P_{\Delta\left(k_{0}\right)}$ on the subset $\Delta\left(k_{0}\right)$. Correspondingly the state

$$
\left|\psi_{\nu}>=\int \psi_{q_{0}}\left(\mathbf{q}_{\nu}\right)\right| \mathbf{q}_{\nu}>d^{3} q_{\nu}
$$

is eigenstate of the projection operator on the subset $\Delta\left(q_{0}\right)\left(E_{q_{0}} \leq E_{k} \leq\right.$ $\left.E_{q_{0}}+\varepsilon E_{q_{0}}, \quad E_{q_{0}}=E_{k_{0}} \nu^{-2}\right)$. The projection operators are defined for any subsets even when their location tend to the infinite part of the spectrum. From this it follows that even when $\nu$ tends to infinity eigenvectors of the projection operator $P_{\Delta\left(q_{0}\right)}$ belong to the Hilbert space $\mathcal{H}$. However, for describing such states we have to make a change the scale by letting $\nu$ to infinity. For any $\nu$ the vectors $\left|\psi_{\nu}\right\rangle$ can be represented as vectors of the Hilbert space $L^{2}\left(M_{\nu}\right)$ of square integrable functions $\psi\left(\mathbf{q}_{\nu}\right)$, where $M_{\nu}$ denotes the momentum-space corresponding to the scale $\nu$. Obviously, for any finite $\nu$ the spaces $L^{2}\left(M_{\nu}\right)$ coincides each with other, since $\psi\left(\mathbf{q}_{\nu}\right)=\psi^{\prime}(\mathbf{k})=\psi(\mathbf{k} / \nu)$. This, of course, is not true for the space $L^{2}\left(M_{\infty}\right)$ of square integrable functions $\psi\left(\mathbf{q}_{i n}\right)$, with $\mathbf{q}_{i n}$ being the momentum in the case when the scale tends to infinity. Since for any $|\mathbf{k}|<\infty$

$$
\begin{gathered}
\lim _{\nu \rightarrow \infty}<\mathbf{k}\left|\psi_{\nu}>=\lim _{\nu \rightarrow \infty} \int \psi\left(\mathbf{q}_{\nu}\right)<\mathbf{k}\right| \mathbf{q}_{\nu}>d^{3} q_{\nu}= \\
=\lim _{\nu \rightarrow \infty} \int \psi\left(\mathbf{q}_{\nu}\right)<\mathbf{q}_{\nu}{ }_{\nu} \mid \mathbf{q}_{\nu}>\nu^{-\frac{3}{2}} d^{3} q_{\nu}=\lim _{\nu \rightarrow \infty} \psi(\mathbf{k} / \nu) \nu^{-\frac{3}{2}}=0,
\end{gathered}
$$

each of the vectors $\mid \psi_{\text {in }}>$ of $L^{2}\left(M_{\infty}\right)$ is orthogonal to all vectors of $L^{2}\left(M_{1}\right)$. Thus the Hilbert space describing the states of the system under study is 
where $\mathcal{H}_{p}$ is the space that can be realized as the space $L^{2}\left(M_{1}\right)$, and $\mathcal{H}_{\text {in }}$ is the space that can be realized as the space $L^{2}\left(M_{\infty}\right)$. The manifold of the physically realizable states, being the domain of $H_{0}$, is dense in $\mathcal{H}_{p}$. On the other hand, the vectors of $\mathcal{H}_{\text {in }}$ represent the states with infinite energy, and are not physically realizable.

Let us now show that the matrix element $<\psi_{\text {in }}^{(2)}|R(t, 0)| \psi_{\text {in }}^{(1)}>$, where $R\left(t, t_{0}\right)$ is defined by $U\left(t, t_{0}\right)=\mathbf{1}+i R\left(t, t_{0}\right)$, are not continuous at $t=0$. From (7) and (8) it follows that the operator $R\left(t, t_{0}\right)$ can be represented in the form

$$
R\left(t, t_{0}\right)=-i \int_{t_{0}}^{t} d t_{2} \int_{t_{0}}^{t_{2}} d t_{1} \exp \left(i H_{0} t_{2}\right) \tilde{T}\left(t_{2}-t_{1}\right) \exp \left(-i H_{0} t_{1}\right) .
$$

By using (53), we can write

$$
\begin{gathered}
<\psi_{\nu}^{(2)}|R(t, 0)| \psi_{\nu}^{(1)}>=-i \int_{0}^{t} d t_{2} \int_{0}^{t_{2}} d t_{1} \int d^{3} k^{\prime} \int d^{3} k \exp \left(i E_{k^{\prime}} t_{2}\right) \times \\
\times \exp \left(-i E_{k} t_{1}\right)<\psi_{\nu}^{(2)}\left|\mathbf{k}^{\prime}><\mathbf{k}^{\prime}\right| \tilde{T}\left(t_{2}-t_{1}\right)|\mathbf{k}><\mathbf{k}| \psi_{\nu}^{(1)}>.
\end{gathered}
$$

Taking into account (25), (40) and (47), and letting $\nu$ to infinity, we get

$$
\begin{gathered}
<\psi_{i n}^{(2)}|R(t, 0)| \psi_{i n}^{(1)}>=\lim _{\nu \rightarrow \infty}<\psi_{\nu}^{(2)}|R(t, 0)| \psi_{\nu}^{(1)}>=-i a_{1}\left|c_{1}\right|^{2} \lim _{\nu \rightarrow \infty} \int_{0}^{t \nu^{2}} d \theta_{2} \times \\
\times \int_{0}^{\theta_{2}} d \theta_{1} \int d^{3} q_{\nu}^{\prime} \int d^{3} q_{\nu} \exp \left(i E_{q_{\nu}^{\prime}} \theta_{2}\right) \exp \left(-i E_{q_{\nu}} \theta_{1}\right) \psi_{2}^{*}\left(\mathbf{q}_{\nu}^{\prime}\right) \psi_{1}\left(\mathbf{q}_{\nu}\right) \times \\
\times \nu^{-1}\left|\nu \mathbf{q}_{\nu}\right|^{-\alpha}\left|\nu \mathbf{q}_{\nu}^{\prime}\right|^{-\alpha}\left(\frac{\theta_{2}-\theta_{1}}{\nu^{2}}\right)^{-\frac{1}{2}-\alpha},
\end{gathered}
$$

where $\theta_{i}=t_{i} \nu^{2}, \psi_{i}\left(\mathbf{q}_{\nu}\right)=<\mathbf{q}_{\nu} \mid \psi_{\nu}^{i}>, i=1,2$. From this it follows that in the limit $\nu \rightarrow \infty$ the matrix elements $<\psi_{\nu}^{(2)}|R(t, 0)| \psi_{\nu}^{(1)}>$ are scale invariant, and we have

$$
\begin{gathered}
<\psi_{i n}^{(2)}|R(t, 0)| \psi_{i n}^{(1)}>=-i a_{1}\left|c_{1}\right|^{2} \int_{0}^{\infty} d \theta_{2} \int_{0}^{\theta_{2}} d \theta_{1} \int d^{3} q^{\prime} \int d^{3} q \exp \left(i E_{q^{\prime}} \theta_{2}\right) \times \\
\times \exp \left(-i E_{q} \theta_{1}\right) \psi_{2}^{*}\left(\mathbf{q}^{\prime}\right) \psi_{1}(\mathbf{q})|\mathbf{q}|^{-\alpha}\left|\mathbf{q}^{\prime}\right|^{-\alpha}\left(\theta_{2}-\theta_{1}\right)^{-\frac{1}{2}-\alpha}
\end{gathered}
$$

Thus the matrix elements $<\psi_{i n}^{(2)}|R(t, 0)| \psi_{\text {in }}^{(1)}>$ are independent of $t$, and do not tend to zero as $t \rightarrow 0$. This means that the amplitudes $<\psi_{\text {in }}^{(2)}|R(t, 0)| \psi_{\text {in }}^{(1)}>$ are not continuous at $t=0$ since, as it follows from the definition, $R(0,0)=0$. From this it follows that the evolution operator (38) is not weakly continuous. On the other hand one can show that for the physically realizable states $\mid \psi_{1}>$ and $\mid \psi_{2}>$ the matrix elements $<\psi_{2}|U(t, 0)| \psi_{1}>$ tend to $<\psi_{2} \mid \psi_{1}>$ as $t \rightarrow 0$ and hence the condition (6) is not violated. Nevertheless, the evolution operator $V(t)=U_{s}(t, 0)$ is not continuous and hence the group of 
that in this case the time evolution of a state vector is not governed by the Schrödinger equation. The cause of this discontinuity is quite obvious. In fact, from the point of view of the states with infinite energy any time interval $\delta t$ is infinite and hence the matrix element $<\psi_{i n}^{(2)}|R(\delta t, 0)| \psi_{i n}^{(1)}>$ must be independent of $\delta t$, i.e. must be constant.

The fact that in principle for describing the evolution of a quantum system during infinitesimal time intervals one has to take into account the intermediate states with infinite energy is a consequence of the principle of uncertainty. In the case of Hamiltonian dynamics, nevertheless, such states give no contributions because the matrix elements $<\mathbf{p}_{2}\left|U\left(t_{2}, t_{1}\right)\right| \mathbf{p}_{1}>$ vanish sufficiently fast when momenta tend to infinity. In this case the space $\mathcal{H}_{\text {in }}$ can be ignored, and the dynamics can be restricted to $\mathcal{H}_{p}$. However, in general case the matrix elements $<\mathbf{p}_{2}\left|U\left(t_{2}, t_{1}\right)\right| \mathbf{p}_{1}>$ may have such a large-momentum behavior that one cannot ignore the space $\mathcal{H}_{\text {in }}$ in describing the dynamics of a quantum system. To better illustrate this point, let us consider the operator $V_{p}(t)=P V(t) P, P$ being the projection operator on the subspace $\mathcal{H}_{p}$. Assume that the group of the operators $V_{p}(t)$ has a self-adjoint infinitesimal generator $A$. Then for $\mid \psi>\in \mathcal{D}(A)$ we have

$$
\frac{V_{p}(t)|\psi>-| \psi>}{t} \underset{t \rightarrow 0}{\rightarrow}-i A \mid \psi>
$$

From this it follows that $A=H_{0}+A_{1}$, with $A_{1}=-\lim _{t \rightarrow 0}\left(\frac{1}{t} \exp \left(-i H_{0} t\right) R(t, 0)\right)$. By using (40) and (53), for $\mid \psi>\in \mathcal{D}\left(H_{0}\right)$ and $|\mathbf{k}|<\infty$, we get

$$
\begin{aligned}
& <\mathbf{k}\left|A_{1}\right| \psi>=i \lim _{t \rightarrow 0}\left(\frac{1}{t} \int_{0}^{t} d t_{2} \int_{0}^{t_{2}} d t_{1} \int d^{3} k_{1} \exp \left[i E_{k}\left(t_{2}-t\right)\right] \times\right. \\
& \left.\times \exp \left(-i E_{k_{1}} t_{1}\right) \varphi^{*}(\mathbf{k}) \varphi\left(\mathbf{k}_{1}\right)<\mathbf{k}\left|\tilde{T}\left(t_{2}-t_{1}\right)\right| \mathbf{k}_{1}>\psi\left(\mathbf{k}_{1}\right)\right)= \\
& =i a_{1}\left|c_{1}\right|^{2} C \varphi^{*}(\mathbf{k}) \lim _{t \rightarrow 0}\left(\frac{1}{t} \int_{0}^{t} d t_{2} \int_{0}^{t_{2}} d t_{1}\left(t_{2}-t_{1}\right)^{-\frac{1}{2}-\alpha}\right)=0
\end{aligned}
$$

where $C=\int d^{3} k_{1} \varphi\left(\mathbf{k}_{1}\right) \psi\left(\mathbf{k}_{1}\right)$. Hence $A=H_{0}$, i.e. the infinitesimal generator of the group of the operators $V_{p}(t)$ is equal to the free Hamiltonian. This means that the dynamics of the system cannot be restricted to the space $\mathcal{H}_{p}$.

It is important to now whether it is possible to find the system at time $t$ in a state $\mid \psi_{i n} \in \mathcal{H}_{i n}$, if initially at time $t_{0}$ the state of the system was physically realizable. The amplitude of this possibility is given by the matrix element $<\psi_{i n}|U(t, 0)| \psi_{1}>$ with $\mid \psi_{1}>\in \mathcal{H}_{p}$. For this matrix element we can write 


$$
\begin{gathered}
\times \exp \left(-i E_{k^{\prime}} t_{1}\right) \psi^{*}(\mathbf{k} / \nu) \nu^{-\frac{3}{2}} \varphi^{*}(\mathbf{k})<\mathbf{k}\left|\tilde{T}\left(t_{2}-t_{1}\right)\right| \mathbf{k}^{\prime}>\varphi\left(\mathbf{k}^{\prime}\right) \psi_{1}\left(\mathbf{k}^{\prime}\right)= \\
=a_{1} c_{1}^{*} \lim _{\nu \rightarrow \infty} \int_{0}^{t \nu^{2}} d \theta_{2} \int_{0}^{\theta_{2}} d \theta_{1} \int d^{3} q_{\nu} \int d^{3} k^{\prime} \exp \left(i E_{q_{\nu}} \theta_{2}\right) \times \\
\times\left(\theta_{2}-\theta_{1}\right)^{-\frac{1}{2}-\alpha}\left|\mathbf{q}_{\nu}\right|^{-\alpha} \psi^{*}\left(\mathbf{q}_{\nu}\right) \psi_{1}\left(\mathbf{k}^{\prime}\right) \nu^{-\frac{3}{2}}=0 .
\end{gathered}
$$

Thus, if at time $t_{0}$ the state of the system was physically realizable, the probability to finding the system at time $t$ in any state $\mid \psi>\in \mathcal{H}_{\text {in }}$ is equal to zero, despite the fact that one cannot ignore the subspace $\mathcal{H}_{i n}$. This means that the states $\mid \psi>\in \mathcal{H}_{i n}$ are not observable. This is not at variance with the fact that one have to take into account the space $\mathcal{H}_{\text {in }}$ in describing the time evolution of the system. Indeed, in the limit $t \rightarrow 0$ all matrix elements $<\psi_{2}|R(t, 0)| \psi_{1}>$ tend to zero, provided that $\mid \psi_{1}>\in \mathcal{H}_{p}$. However, for $\mid \psi_{2}>\in \mathcal{H}_{p}$ the matrix elements $<\psi_{2}|R(t, 0)| \psi_{1}>$ vanishes faster than $<\psi_{\text {in }}|R(t, 0)| \psi_{1}>$ when $t \rightarrow 0$. This results in the fact that, as we have seen, the intermediate states belonging to $\mathcal{H}_{\text {in }}$ are responsible for validity of the composition law (3) for infinitesimal time intervals. Thus these states play the key role in the time evolution of the system in the infinitesimal neighborhood of the point $t=0$.

To clarify this point, note the following. The concept of nonlocal-in-time potentials were first introduced within the optical-potential model. The optical potentials are introduced in the case when only state vectors belonging some subspace of the Hilbert space $\mathcal{A}$ are included explicitly in the description of the time evolution of a quantum system. Such potentials which globally accounts for the coupling between the subspace $\mathcal{A}$ and its complementary part $\mathcal{B}$ are nonlocal in time, and depend on the history of the system. The nonlocal form of the optical potentials is an expression of the loss of probability from the subspace $\mathcal{A}$, i.e. of the fact that the evolution operator defined on $\mathcal{A}$ is not unitary. The nonlocality in time of effective interaction operator in the case, when the dynamics of a quantum system is restricted to some subspace of the Hilbert space, is a consequence of the coupling between this subspace and its complementary part. This clarifies the fact that in our model the interaction operator becomes nonlocal in time in the case $\alpha<\frac{1}{2}$ when the ultraviolet behavior of the form factors is "bad", and one cannot ignore the subspace $\mathcal{H}_{\text {in }}$ of states with infinite energy. In this case we also have to deal with two subspaces: $\mathcal{H}_{p}$ and its complementary part $\mathcal{H}_{i n}$. However, in our model the coupling between these subspaces does not lead to the loss of probability from the subspace $\mathcal{H}_{p}$ : The evolution operator $P U\left(t, t_{0}\right) P, P$ being the orthogonal projection on $\mathcal{H}_{p}$, is unitary. The remarkable feature of the GQD is that it allows one to restrict the description of the unitary 
account the coupling between this space and $\mathcal{H}_{i n}$. In fact, as we have seen, the space $\mathcal{H}_{\text {in }}$ manifests itself only in the behavior of the evolution operator $U\left(t, t_{0}\right)$ in the infinitesimal neighborhood of the point $t=t_{0}$. On the other hand, the physical meaning of the generalized interaction operator $H_{\text {int }}\left(t_{2}, t_{1}\right)$ is that it determines the evolution of the system in this neighborhood. In order that $H_{\text {int }}\left(t_{2}, t_{1}\right)$ contain all needed dynamical information, in the limit $t_{2} \rightarrow t_{1}$ the operator $U_{H}\left(t, t_{0}\right)=\int_{t_{0}}^{t} d t_{2} \int_{t_{0}}^{t_{2}} d t_{1} H_{\text {int }}\left(t_{2}, t_{1}\right)$ must be close enough to the true evolution operator $U\left(t, t_{0}\right)$. Thus the solution of the evolution problem is divided in two stages: The first step is to determine the operator $H_{\text {int }}\left(t_{2}, t_{1}\right)$ describing the time evolution in the infinitesimal neighborhood of the point $t=t_{0}$, where the states belonging to $\mathcal{H}_{\text {in }}$ play an important role, and the next step is to describe the dynamics restricted to $\mathcal{H}_{p}$ starting with the above generalized interaction operator that takes into account the coupling between the spaces $\mathcal{H}_{p}$ and $\mathcal{H}_{i n}$.

To illustrate this point, let us come back to our model. From (29) it follows that the solution of Eq.(27) can be represented in the form

$$
t(z)=\lim _{a \rightarrow-\infty} g_{a}\left(1+g_{a} J(z, a)\right)^{-1},
$$

where

$$
J\left(z_{1}, z_{2}\right)=\left(z_{1}-z_{2}\right) \int d^{3} k \frac{|\varphi(\mathbf{k})|^{2}}{\left(z_{1}-E_{k}\right)\left(z_{2}-E_{k}\right)} .
$$

In the case $\alpha<\frac{1}{2}, J(z, a)$ tends to infinity like $|a|^{\frac{1}{2}-\alpha}$ when $a \rightarrow-\infty$. From this it follows that, for the solution $t(z)$ to be nonzero, $g_{a}$ must decrease like $|a|^{\alpha-\frac{1}{2}}$ as $a \rightarrow-\infty$. Thus the leader term of $g_{a}$ in the limit $a \rightarrow-\infty$ must be of the form $b_{1}|a|^{-\frac{1}{2}+\alpha}$, where the parameter $b_{1}$ is completely determined by the above requirement of nontriviality of the solution. Indeed, by using the identity $J\left(z_{1}, z_{2}\right)=J\left(z_{1}, z\right)+J\left(z, z_{2}\right)$, we can rewrite (59) in the form

$$
t(z)=\lim _{a \rightarrow-\infty} g_{a}\left(1+g_{a} J(0, a)+g_{a} J(z, 0)\right)^{-1} .
$$

From this formula we see that $g_{a} J(0, a)$ must tend to one as $a \rightarrow-\infty$, since in this limit $g_{a}$ tends to zero. Hence we have

$$
\begin{gathered}
b_{1}=-\lim _{\nu \rightarrow \infty}\left(\left|a_{0}\right|^{\frac{1}{2}+\alpha} \nu^{2 \alpha} \int d^{3} q_{\nu} \frac{\left|\varphi\left(\nu \mathbf{q}_{\nu}\right)\right|^{2}}{\left(E_{q_{\nu}}-a_{0}\right) E_{q_{\nu}}}\right)^{-1}= \\
=-\left(\left|a_{0}\right|^{\frac{1}{2}+\alpha}\left|c_{1}\right|^{2} \int d^{3} q_{\nu}\left|\psi\left(\mathbf{q}_{i n}\right)\right|^{2}\right)^{-1}
\end{gathered}
$$

where $a=a_{0} \nu^{2}, a_{0} \in(-\infty, 0), \psi\left(\mathbf{q}_{i n}\right)=\left|\mathbf{q}_{i n}\right|^{-\alpha}\left(E_{q_{i n}}-a_{0}\right)^{-\frac{1}{2}} E_{q_{i n}}^{-\frac{1}{2}}$. Thus the 
$L^{2}\left(M_{\infty}\right)$ being the realization of the space $\mathcal{H}_{i n}$. From $(60)$ it also follows that the next order term in the asymptotic behavior of $g_{a}$ must be of the form $b_{2}|a|^{-1+2 \alpha}$, where the freedom of choice of the parameter $b_{2}$ is bounded only by the requirement of unitarity of the resulting evolution operator. Thus, in the limit $a \rightarrow-\infty$ the function $t(a)=g_{a}$ behaves like $b_{1}|a|^{-\frac{1}{2}+\alpha}+b_{2}|a|^{-1+2 \alpha}$, and hence the generalized interaction operator must be of the form (36), and the corresponding solution of Eq.(17) is as follows:

$$
<\mathbf{p}_{2}|T(z)| \mathbf{p}_{1}>=-\varphi^{*}\left(\mathbf{p}_{2}\right) \varphi\left(\mathbf{p}_{1}\right)\left(b_{2} b_{1}^{-2}+\int d^{3} k \frac{z|\varphi(\mathbf{k})|^{2}}{\left(z-E_{k}\right) E_{k}}\right)^{-1} .
$$

It is easy to verify that (62) is equivalent to the above obtained expression (32). Here we are dealing only with the functions belonging to the space $L^{2}\left(M_{1}\right)$. Thus after specifying the form of the generalized interaction operator the description of the dynamics can be restricted to the space of the physically realizable states. Note that in the case $\alpha>\frac{1}{2}$ such problems do not arise. Indeed, in this case $g_{a}$ in (29) must tend to $\lambda$ being an arbitrary real constant. Thus, in this case we directly get the expression (32), which is written in terms of functions belonging to the space $L^{2}\left(M_{1}\right)$.

By the example of the exactly solvable model we have shown that nonlocality in time of the interaction generating the unitary dynamics of a quantum system should be associated with the existence of the space $\mathcal{H}_{\text {in }}$ of unobservable states that has to be taken into account in describing the time evolution of the system. In our model the space $\mathcal{H}_{p}$ may be considered as the space of two-nucleon states. The quark structure of the nucleons being in such states does not directly manifest itself since, if the system is in the state $\mid \psi>\in \mathcal{H}_{p}$, the probability of finding free quarks, when a measurement is performed, is equal to zero. In this case, the space $\mathcal{H}_{i n}$ may be considered as the space of states in which the quark structure of nucleons manifests itself. The spaces $\mathcal{H}_{p}$ and $\mathcal{H}_{i n}$ have the same structure. In the general case the structure of these spaces may be different. For example, in the case of hadron dynamics the space $\mathcal{H}_{i n}$ of states corresponding to infinite momentum scale, i.e. to infinitesimal length scale, may describe any states of quarks and gluons, while due to confinement the space $\mathcal{H}_{p}$ describes only observable hadron states (color singlets).

Note in this connection that, as we have stated, there are two different relevant scales in the physics of the strong interaction, and their separation is so significant that the length scale of processes in which quarks and gluons are relevant degrees of freedom can be regarded as infinitesimal compared to the relevant length scale of the low-energy hadron physics. Indeed, these 
intervals. From this in turn it follows that the relevant momentum scale of states in which quarks and gluons can manifest themselves must be infinitely large. Nevertheless these degrees of freedom can have significant effects on hadron dynamics, and for its describing one has to take into account the coupling between the space of hadron states and the space describing states of quark and gluons, and, as usual, this coupling should result in nonlocality in time of hadron interactions. However, in contrast with ordinary open quantum systems, due to confinement this nonlocality must not lead to a loss of probability from the hadron system: The probability of finding the system in a state containing quarks and gluons, when a measurement is performed, is equal to zero. Nevertheless one cannot ignore the subspace describing such states. As we have seen, it is just the case when one has to go beyond Hamiltonian dynamics. The remarkable feature of the GQD is that it provides the extension of quantum dynamics to this case. This opens new possibilities to find the link between the quark-gluon dynamics and low-energy hadron dynamics. We can try to construct the operator of hadron interactions by using the quark-gluon dynamics, and then use it for describing hadron dynamics. As we will show in the next section, for constructing this operator one can also use some quark models.

The essential lesson we have learned from the previous analysis is that the existence of the quark and gluon degrees of freedom confined within hadrons gives rise to the peculiar dynamical situation: In the large-momentum limit the matrix elements of the evolution operator do not decrease so rapidly as it is required by ordinary quantum mechanics, and this results in the above-mentioned lack of continuity of the evolution operator, and correspondingly in the fact that hadron dynamics is not governed by the Schrödinger equation. More precisely, the above are retardation effects from quark confinement on hadron dynamics, since the existence of some external degrees of freedom in itself cannot have such effects on the dynamics of a quantum system. As we have seen, the retardation effects from quark confinement can be very essential. For example, as we will show in Sec.VI, these effects give rise to an anomalous off-shell behavior of the two-nucleon amplitudes.

\section{Generalized Interaction Operator and Quark Models}

As we have shown, quark-gluon retardation must result in nonlocality in time of hadron interactions. From this it follows that low-energy hadron dynamics 
generalized interaction operator describing the history of the hadron system in an infinitesimal time interval. Here we mean the dynamical system whose states correspond to a complete set of hadronic observables. In the case of low-energy hadron dynamics, we can consider as infinitesimal such time intervals, for which only the processes being the fundamental at the hadronic level, for example, the processes being described by the baryon-baryon-meson and four-baryon vertices, are relevant. The generalized interaction operator $H_{\text {int }}\left(t_{2}, t_{1}\right)$ represents the history of the hadron system in infinitesimal time intervals, during which the quark and gluon degrees of freedom can manifest themselves, and in principle it should be extracted from QCD. However, a nonperturbative treatment of $\mathrm{QCD}$ is not possible until now, and for constructing the generalized interaction operator we have to restrict ourselves to using some quark models.

Let us now show that the dynamics of nucleons with the internal structure described by a constituent quark model is governed by the equation of motion (9) with a nonlocal-in-time operator $H_{\text {int }}\left(t_{2}, t_{1}\right)$. In constituent quark models nonstrange and strange baryons are considered to be clusters made of three valence quarks. The Hamiltonian used in these models has the following form:

$$
H=\sum_{i} \frac{p_{i}^{2}}{2 m_{q}}-T_{c . m .}+V_{c o n}\left(\mathbf{r}_{i j}\right)+V_{q q}\left(\mathbf{r}_{i j}\right),
$$

where $\mathbf{r}_{i j}$ represents the interquark distance, $T_{c . m}$. is the kinetic energy of the center of mass motion, $V_{q q}\left(\mathbf{r}_{i j}\right)$ is the one-gluon exchange (OGE) potential, and $V_{c o n}\left(\mathbf{r}_{i j}\right)$ is the confinement potential

$$
V_{\text {con }}\left(\mathbf{r}_{i j}\right)=-a_{c} \boldsymbol{\Lambda}_{i} \boldsymbol{\Lambda}_{j} \mathbf{r}_{i j}^{2}
$$

Here $a_{c}$ is the confinement strength, and $\boldsymbol{\Lambda}_{i}$ are the $\mathrm{SU}(3)$ color matrices. In the models baryon states are represented by the wave functions of a three-quark oscillator with the Hamiltonian (63). As is well known, such models provide a very satisfactory description of the baryon spectra. In order to take into account the long- and intermediate- range part of the interaction, in some models in addition to the OGE interaction, the quarks belonging to different three-quark clusters interact via scalar and pseudoscalar meson exchange.

For solving the two-nucleon problem within a constituent quark model, one has to consider the dynamics of the six-quark system. Due to confinement potential the six-quark system can be only in the two-baryon states $\mid \psi ; 2 B>$ or in the baglike states $|\psi ; 6 q\rangle$. However, for large time intervals the system 
$\tau_{c}$, during which the system can be in baglike states, is very small and depends on the confinement length $\lambda_{c}$. For the time intervals large compared with $\tau_{c}$, the matrix elements $\left\langle\psi_{2} ; 6 q\left|U\left(t, t_{0}\right)\right| \psi_{1}>\right.$ describing the probability to find the system in the state $\mid \psi_{2} ; 6 q>$ at time $t$ are neglectable, and we can write

$$
<\psi_{2} ; 6 q\left|U\left(t, t_{0}\right)\right| \psi_{1}>=0 .
$$

From this it follows that for large time intervals the operator $U_{2 B}\left(t, t_{0}\right)$ obtained by projecting $U_{2 B}\left(t, t_{0}\right)=P_{2 B} U\left(t, t_{0}\right) P_{2 B}$, where $P_{2 B}$ is the projection operator on the subspace $\mathcal{H}_{2 B}$, can be considered to be unitary.

Let us now show that the representation (7) is valid for this operator. By using the composition law (3) for the evolution operator $U\left(t, t_{0}\right)$, we can write

$$
U\left(t, t_{0}\right)=\prod_{j=1}^{N} U\left(t_{j}, t_{j-1}\right),
$$

where $t_{j}=t_{0}+j \xi, \xi=\left(t-t_{0}\right) / N, t_{N}=t$. Within Hamiltonian dynamics the evolution operator $U\left(t_{j+1}, t_{j}\right)$ has the form

$$
U\left(t_{j}, t_{j-1}\right)=\exp \left(i H_{0} t_{j}\right) \exp \left[-i H\left(t_{j}-t_{j-1}\right)\right] \exp \left(-i H_{0} t_{j-1}\right) .
$$

In the limit $\xi \rightarrow 0$ we can write

$$
U\left(t_{j}, t_{j-1}\right) \approx \exp \left(i H_{0} t_{j+1}\right)(\mathbf{1}-i \xi H) \exp \left(-i H_{0} t_{j-1}\right) .
$$

By using (65), the evolution operator can be represented in the form

$$
U\left(t, t_{0}\right)=\lim _{\xi \rightarrow 0} \prod_{j=1}^{N} \exp \left(i H_{0} t_{j}\right)(\mathbf{1}-i \xi H) \exp \left(-i H_{0} t_{j-1}\right) .
$$

It is easy to verify that (68) can be rewritten in the form

$$
U\left(t, t_{0}\right)=\mathbf{1}-\lim _{\xi \rightarrow 0} \sum_{l=1}^{N}\left(i \xi H_{I}\left(t_{l}\right)+\sum_{k=1}^{l} \xi^{2} H_{I}\left(t_{l}\right) U\left(t_{l}, t_{k}\right) H_{I}\left(t_{k}\right)\right) .
$$

Taking the limit $\xi \rightarrow 0$, from (69), we get

$$
U\left(t, t_{0}\right)=\mathbf{1}-\int_{t_{0}}^{t} d t_{2} \int_{t_{0}}^{t_{2}} d t_{1}\left(2 i \delta\left(t_{2}-t_{1}\right) H_{I}\left(t_{1}\right)+H_{I}\left(t_{2}\right) U\left(t_{2}, t_{1}\right) H_{I}\left(t_{1}\right)\right) .
$$

Thus, within Hamiltonian dynamics, the evolution operator can be represented in the form (7) with the following operator $\tilde{S}\left(t_{2}, t_{1}\right)$ :

$$
\tilde{S}\left(t_{2}, t_{1}\right)=-2 i \delta\left(t_{2}-t_{1}\right) H_{I}\left(t_{1}\right)-H_{I}\left(t_{2}\right) U\left(t_{2}, t_{1}\right) H_{I}\left(t_{1}\right) .
$$

Correspondingly, for the operator $U_{2 B}\left(t, t_{0}\right)$ defined on the two-baryon space $\mathcal{H}_{2 B}$ we can write 


$$
+\int_{t_{0}}^{t} d t_{2} \int_{t_{0}}^{t_{2}} d t_{1}<\psi_{2}, \beta\left|\tilde{S}_{2 B}\left(t_{2}, t_{1}\right)\right| \psi_{1}, \alpha>
$$

where

$$
\begin{aligned}
<\psi_{2}, \beta \mid & \tilde{S}_{2 B}\left(t_{2}, t_{1}\right)\left|\psi_{1}, \alpha>=<\psi_{2}, \beta\right| P_{2 B} \tilde{S}\left(t_{2}, t_{1}\right) P_{2 B} \mid \psi_{1}, \alpha>= \\
= & -<\psi_{2}, \beta \mid P_{2 B} \exp \left(i H_{0}^{(\beta)} t_{2}\right)\left\{2 i \delta\left(t_{2}-t_{1}\right) H_{I}^{(\alpha)}+\right. \\
& \left.+H_{I}^{(\beta)} U_{s}\left(t_{2}, t_{1}\right) H_{I}^{(\alpha)}\right\} \exp \left(-i H_{0}^{(\alpha)} t_{1}\right) P_{2 B} \mid \psi_{1}, \alpha>.
\end{aligned}
$$

Here we use the interaction picture in which the interaction in the baryon clusters is included into the "free" Hamiltonian $H_{0}^{(\alpha)}$ of the corresponding two-baryon channel $H=H_{0}^{(\alpha)}+H_{I}^{(\alpha)}$, and $|\psi, \alpha\rangle$ is the vector belonging to the channel subspaces $\mathcal{H}_{\alpha}$.

We have shown that the operator $U_{2 B}\left(t, t_{0}\right)$ can be represented in the form (7), where the operator $\tilde{S}\left(t_{2}, t_{1}\right)$ is given by (73). As has been shown in Ref.[21], for the operator $U_{2 B}\left(t, t_{0}\right)$ having the form (7) to be unitary for any times $t$ and $t_{0}$, the operator $\tilde{S}_{2 B}\left(t_{2}, t_{1}\right)$ must satisfy the equation

$$
\left(t_{2}-t_{1}\right) \tilde{S}_{2 B}\left(t_{2}, t_{1}\right)=\int_{t_{1}}^{t_{2}} d t_{4} \int_{t_{1}}^{t_{4}} d t_{3}\left(t_{4}-t_{3}\right) \tilde{S}_{2 B}\left(t_{2}, t_{4}\right) \tilde{S}_{2 B}\left(t_{3}, t_{1}\right) .
$$

However, the operator $U_{2 B}\left(t, t_{0}\right)$ can be considered as a unitary operator only for time intervals $t-t_{0}$ large compared with $\tau_{c}$. Correspondingly, the operator $\tilde{S}_{2 B}\left(t_{2}, t_{1}\right)$ satisfies the equation (74) only for time intervals $t_{2}-t_{1}>>\tau_{c}$. On the other hand, due to confinement time intervals, which are relevant for the description of low-energy nucleon dynamics, are large compared with $\tau_{c}$ (here we are dealing with two separated length scales). From this it follows that the low-energy dynamics of the two-nucleon system predetermined by a constituent quark model is governed by Eq.(9).

Up to now we restricted ourselves to the consideration of the two-nucleon dynamics. On the other hand, there are time intervals large, compared with $\tau_{c}$, that are much smaller than the time intervals relevant for the many-nucleon interactions. Hence, for such "infinitesimal" intervals of time the operator $\tilde{S}_{N}\left(t_{2}, t_{1}\right)$, which is the two-nucleon operator on the many-nucleon Hilbert space, satisfies Eq.(9). For this reason, the operator $\tilde{S}_{N}\left(t_{2}, t_{1}\right)$ related to the operator $\tilde{S}_{2 B}\left(t_{2}, t_{1}\right)$ in the ordinary way can be used as the generalized interaction operator generating the dynamics of the many-nucleon system

$$
H_{\text {int }}\left(t_{2}, t_{1}\right)=\tilde{S}_{N}\left(t_{2}, t_{1}\right) .
$$

From (73) it follows that this operator can be represented in the form (22), where the long- and intermediate-range parts of $H_{I}(t)$ present the 
in the six-quark Hamiltonian, and its short-range part is a potential arising due to the one-gluon-exchange potential in this Hamiltonian. The nonlocal term $H_{\text {non }}\left(t_{2}, t_{1}\right)$ describes the contributions from processes in which during some time intervals retardation from quark confinement can take place. The contribution from such processes cannot be neglected, and the effective $N N$ interaction extracted from a constituent quark model must contain the nonlocal-in-time term leading to the above-mentioned dynamical situation. This is an expression of the fact that there are some effects, related to the short-range $N N$ system, that cannot be described in terms of the $N N$ potentials (see, for example, [8]).

\section{VI.Nonlocality in time of the NN interaction and an anomalous off-shell behavior of two-nucleon amplitudes.}

The results given in Sec.IV can be used for constructing models which allows one to take into account the quark-gluon retardation effects in describing the NN interaction. As the first step in this direction, we can generalize the ordinary separable-potential model that is widely used in nuclear physics. At the present time, for two-nucleon separable potentials it is usually used the Yamaguchi and Tabakin form factors $[27,28]$ which in spin-triplet and spin-singlet channels are of the form

$$
\begin{gathered}
g_{Y}(\mathbf{p})=\frac{1}{p^{2}+\beta^{2}}, \\
g_{T}(\mathbf{p})=\frac{\left(p^{2}+\nu\right)}{\left(p^{2}+\eta\right)} \frac{\left(q_{c}^{2}-p^{2}\right)}{\left(p^{2}+\gamma^{2}\right)^{k}},
\end{gathered}
$$

where $g_{Y}(p)$ and $g_{T}(p)$ are respectively Yamaguchi and Tabakin form factors, and $p=|\mathbf{p}|$. Such form factors with the parameters given in Ref. [29] have been recently used by Rupp and Tjon [30] and Adnikari and Tomio [31]. In Refs.[31], for example, the Tabakin form factor has been used in one of the nucleon-nucleon spin channels and Yamaguchi in the other.

As it follows from the analysis of Sec.IV, the separable potentials cannot take into account the quark-gluon retardation in describing the NN interaction, since in this case the NN interaction is instantaneous. For the interaction to be nonlocal in time, in the large-momentum limit the form factor $\varphi(\mathbf{p})$ must behave like (25) with $0<\alpha<\frac{1}{2}$, i.e. must have the asymptotic behavior that in the case of the separable potentials leads to the ultraviolet divergences. In this case the generalized interaction operator must 
the coupling between the space $\mathcal{H}_{p}$ of the observable states of the system under study and the space $\mathcal{H}_{\text {in }}$ of unobservable states manifests itself only in the asymptotic behavior of the form factor $\varphi(\mathbf{p})$ in the limit $|\mathbf{p}| \rightarrow \infty$, and in the behavior of the $H_{\text {int }}^{(s)}(\tau)$ in the infinitesimal neighborhood of the point $\tau=0$. Note that in the case of the NN interaction the space $\mathcal{H}_{p}$ should be considered as the space $\mathcal{H}_{N}$ of the two-nucleon states, and $\mathcal{H}_{i n}$ as the space $\mathcal{H}_{q}$ of states, in which the quark structure of nucleons manifests itself. Since the parameter $a_{1}$ in (36) is completely determined by the parameters $\alpha$ and $c_{1}$ characterizing the asymptotic behavior of the form factor $\varphi(\mathbf{p})$, we have only three free parameters $\alpha, c_{1}$, and $a_{2}$ responsible for the coupling between the spaces $\mathcal{H}_{N}$ and $\mathcal{H}_{q}$. However, this true only in the case of the rank-one separable approximation. In this case these three parameters can be determined, for example, by fitting the NN phase shifts. In a more general case, when we cannot restrict ourselves to this approximation, we have much more parameters responsible for the coupling between the spaces $\mathcal{H}_{N}$ and $\mathcal{H}_{q}$, and these parameters should be extracted from quark-gluon dynamics. Let us consider the rank-one separable model of the $N N$ interaction. Let the form factor $\varphi(\mathbf{p})$ in (24) be of the form

$$
\varphi(\mathbf{p})=\chi(\mathbf{p})+c_{2} g_{Y}(\mathbf{p}),
$$

with

$$
\chi(\mathbf{p})=\frac{c_{1}}{\left(d^{2}+\mathbf{p}^{2}\right)^{\frac{\alpha}{2}}}, \quad 0<\alpha<\frac{1}{2},
$$

where $d, c_{1}$ and $c_{2}$ are some constants. Since $\alpha<\frac{1}{2}$, the generalized interaction operator must be of the form (36), and correspondingly the T matrix is given by (37). To have a bound-state corresponding to the deuteron binding energy $E_{d}=2.225 \mathrm{MeV}$, in the case of the $n p$ scattering, the following condition must be satisfied:

$$
-b_{2}+b_{1} E_{d}^{\frac{1}{2}-\alpha}+b_{1}^{2} M\left(-E_{d}\right)=0 .
$$

Taking into account (79) and fitting the $N N$ phase shifts in the range 0-350 $\mathrm{MeV}$, we have obtained the parameters of the interaction operator given by (36). The results for the $\mathrm{S}$ wave phase shifts are shown in Figs.(1-3). Values for the corresponding constants are given in Table 1. It can be seen from Figs.(1-3) that our model yields nucleon-nucleon phase shifts in good agreement with experiment despite the fact that the interaction operator given by (36) is rank-one separable. The phase shifts in ${ }^{1} S_{0}$ channels change sign as well as in the case of Tabakin-type potentials. At the same time, our model has more freedom in fitting data than the separable-potential model.

In contrast with other phenomenological models, the form of the 
retardation effects in describing the $N N$ interaction. The nonlocal interaction operator given by (36) describes the history of the two-nucleon system in infinitesimal time intervals during which the quark and gluon degrees of freedom can manifest themselves. Being exactly solvable, our model can be used for investigating some effects of quark-gluon retardation on low-energy nucleon dynamics. As we have shown, there is the correspondence between the form of the generalized interaction operator and the large-momentum behavior of the matrix elements of the evolution operator. In the nonlocal case, these matrix elements as functions of momenta do not tend to zero at infinity so rapidly as it is required by ordinary quantum mechanics, and within the Hamiltonian formalism this leads to the ultraviolet divergences. Correspondingly in the nonlocal case the two-nucleon amplitudes $<\mathbf{p}_{2}|T(z)| \mathbf{p}_{1}>$ also do not decrease when $\left|\mathbf{p}_{i}\right| \rightarrow \infty$ as it is required by Hamiltonian dynamics. Such a large-momentum behavior of the two-nucleon amplitudes is a consequence of the fact that in the nonlocal case the operator $H_{\text {non }}\left(t_{2}, t_{1}\right)$ is not zero and must satisfy Eq.(23). Another consequence of nonlocality in time of the $N N$ interaction is that for fixed momenta $\mathbf{p}_{1}$ and $\mathbf{p}_{2}$ the matrix elements $<\mathbf{p}_{2}|T(z)| \mathbf{p}_{1}>$ tend to zero as $z \rightarrow-\infty$, while, in the local case, they tend to $<\mathbf{p}_{2}|V| \mathbf{p}_{1}>$ in this limit. To illustrate this, we present in Fig.4 the off-shell behavior $<\mathbf{p}_{2}|T(z)| \mathbf{p}_{1}>$ in the limit $z \rightarrow-\infty$. Thus, nonlocality in time of the $N N$ interaction caused by quark-gluon retardation effects gives rise to an anomalous off-shell behavior of the two-nucleon amplitudes. The off-shell properties of the amplitudes for the ordinary interaction operator and the operator containing the nonlocal term are qualitatively different. This is true even when the two interaction operators have approximately the same phase shifts. Such a large variation in the off-shell behavior of the amplitudes, even when the interaction operators are identical on-shell, can have significant effects on three- and many-body results [33]. This gives reason to expect that the anomalous off-shell behavior of the two-nucleon amplitudes can also have significant effects on nucleon matter properties. From this in turn it follows that the quark and gluon degrees of freedom can play a significant role in low-energy nucleon dynamics.

\section{Conclusion}

As we have shown, the GQD provides the extension of Hamiltonian dynamics which can describe the evolution of a quantum system with confined degrees of freedom that cannot be associated with some observables. We have shown 
deal with two infinitely separated momentum (or length) scales, one of which is relevant for the observable degrees of freedom, and another is relevant for unobservable ones. This gives rise to a peculiar dynamical situation: In the large-momentum limit the matrix elements of the evolution operator do not decrease so rapidly as it required by ordinary quantum mechanics, and this results in the lack of continuity of the evolution operator, and correspondingly in the fact that the evolution of the system is not governed by the Schrödinger equation. The advantage of the GQD consists in the fact it permits the solution of the evolution problem in this case. As we have shown, this open new possibilities for describing low-energy hadron dynamics. This dynamics can be described in terms of the hadronic degrees of freedom, while the generalized interaction operator accounts for the coupling between the space describing hadron states and the space describing the states, in which the quark structure of hadrons manifests itself. This operator can be extracted from quark-gluon dynamics, or can be constructed by some quark models. This has been illustrated by the example of the dynamics of nucleons with the internal structure described by a constituent quark model. This dynamics has been shown to be governed by the generalized equation of motion (9) with nonlocal interaction operator $H_{\text {int }}\left(t_{2}, t_{1}\right)$ given by $(75)$.

We have constructed a model which is an extension of the ordinary separable-potential model to the case where the $N N$ interaction is nonlocal in time, and the form of the interaction operator takes into account retardation effects from quark confinement. Despite its simplicity the model yields the NN phase shifts in good agreement with experiment. At the same time, the nonlocal interaction operator constructed within the model can be used for describing the SR part of the NN interaction.

The main conclusion that may be drawn from the present work is that quark-gluon retardation can have significant effects on low-energy hadron dynamics We have shown that one of the important consequences of quarkgluon retardation effects is the anomalous off-shell behavior of the two-nucleon amplitudes: The elements of the two-nucleon T matrix as functions of momenta have the large-momentum behavior which leads to the ultraviolet divergences in the Hamiltonian formalism. Since the off-shell properties of two-particle amplitudes are crucial for the three- and many-body results, the anomalous off-shell behavior of the two-nucleon amplitudes can have substantial effects on the dynamics of many nucleon systems, and on the properties of nuclear matter. Because such an anomalous behavior of the two-nucleon amplitudes takes place only in the case, when the interaction operator (22) contains the nonlocal term $H_{\text {non }}\left(t_{2}, t_{1}\right)$ that account for the coupling with the space 
describing states, in which the quark structure of hadrons manifests itself, the above means that one cannot ignore this short-ranged term in constructing effective operators of the NN interaction. This is especially important for solving the many-nucleon problem.

\section{Acknowledgments}

We would like to thank W. Greiner, I. N. Mishustin and W. Scheid for helpful discussions and valuable comments. R.Kh.G. would like to acknowledge the hospitality of Institut für Theoretische Physik der Justus-Leibig-Universität,Giessen, where part of this work was completed. 


\section{References}

[1] S. Pal, M. Hanauske, I. Zakout, H. Stöcker, and W. Greiner, Phys. Rev. C 60, 015802 (1999).

[2] A. Faessler, F. Fernandez, G. Lübeck and K. Shimizu, Phys. Lett. B 112, 201 (1982); Nucl. Phys. A402, 555 (1983).

[3] K. Bräuer, Amand Faessler, F. Fernandez and K.Shimizu, Z. Phys. A 320, 609 (1985).

[4] M. Oka and K. Yazaki, Phys. Lett. B 90 (1980) 41; Nucl. Phys. A402, 477 (1983).

[5] D.R. Entem, A.I. Machvariani, A. Valcarce, A.J. Buchmann, Amand Faessler and F. Fernandez, Nucl. Phys. A602, 308 (1996).

[6] V.I. Kukulin, V.N. Pomerantsev, and A. Faessler, Phys. Rev. C 593021 (1999).

[7] Yu.A. Simonov, Nucl. Phys. A463 231 (1984).

[8] Fl. Stancu, S. Pepin, and Ya. Glosman, Phys. Rev. C 56, 2779 (1997).

[9] D. Bartz and Fl. Stancu, Phys. Rev. C 591756 (1999).

[10] C.-F. Qiao, H.-W. Huang, and K.-T. Chao, Phys. Rev. D 54, 2273 (1996); Phys. Rev. D 60094004 (1999).

[11] R. Machleidt, K. Holinde and Ch. Elster, Phys. Rep. 149 No.1, 1 (1987).

[12] A. D. Lahiff and I. R. Afnan, Phys. Rev. C 56, 2387 (1997).

[13] Yu.S. Kalashnikova, I.M.Narodetsky, and V.P.Yurov, Yad. Fiz., 49, 632 (1989).

[14] Yu.A.Simonov, Phys. Lett. B 107, 1 (1981).

[15] A.G. Baryshnikov, L.D.Blokhintsev, I.M.Narodetsky, and D.A.Savin, Yad. Fiz. 48, 1273 (1988).

[16] A.N. Safronov, Teor. Mat. Fiz. 89, 420 (1991); Yad. Fiz., 57, 208 (1994).

[17] Yu.A. Kuperin, K.A. Makarov, and S.P.Merkuriev, Teor.Mat.Fiz., 75, 431 (1988); 76, 242 (1989). 
[19] M. Orlowski, Helv.Phys.Acta. 56, 1053 (1983).

[20] B.O. Kerbikov, Yad. Fiz. 41, 725 (1985); Teor. Mat. Fiz. 65, 379 (1985).

[21] R.Kh. Gainutdinov, J. Phys. A: Math. Gen. 32, 5657 (1999).

[22] R.P. Feynman, Rev. Mod. Phys. 20, 367 (1948).

[23] R.P. Feynman and A.R. Hibbs, Quantum Mechanics and Path Integrals, (McGraw-Hill, New York, 1965).

[24] R. Kh. Gainutdinov and A.A.Mutygullina, Yad. Fiz. 60, 938 (1997) [Physics of Atomic Nuclei, 60, 841 (1997)].

[25] R. Kh. Gainutdinov and A.A.Mutygullina, Yad. Fiz. 62, 2061 (1999) [Physics of Atomic Nuclei, 62, 1905 (1999)].

[26] R.F.Streater and A.S.Wightman, PCT, Spin and Statistics, And All That, (W.A.Benjamin, New York, 1964).

[27] Y.Yamaguchi, Phys. Rev. 95, 1635 (1954).

[28] F.Tabakin, Phys. Rev. 174, 1208 (1968).

[29] S.K.Adhikari and L.Tomio, Ann. Phys. (N.Y.) 235, 103 (1994).

[30] G.Rupp and J.A.Tjon, Phys.Rev. C 37, 1729 (1988).

[31] S.K.Adhikari and L.Tomio, Phys.Rev. C 51, 70 (1995).

[32] V. G. J. Stoks, R. A. M. Klomp, M. C. M. Rentmeester, and J. J. de Swart, Phys. Rev. C 48, 792 (1993).

[33] R. Machleidt, F. Sammarruca, and Y. Song, Phys. Rev. C 53, 1483 (1996). 
TABLE 1 . The parameters of the interaction operator obtained by fitting the NN date, $\rho=1 \mathrm{MeV}^{-1}$.

\begin{tabular}{|c|c|c|c|c|c|c|}
\hline partial wave & $\alpha$ & $\lambda$ & $b \cdot \rho$ & $d \cdot \rho$ & $c_{1} \cdot \rho^{\alpha-1}$ & $b_{2} \cdot \rho^{1-2 \alpha}$ \\
\hline${ }^{3} S_{1}(n p)$ & 0.499 & 200.2 & 433.8 & 766.2 & 0.015 & $7.538 \cdot 10^{-4}$ \\
\hline${ }^{1} S_{0}(n p)$ & 0.499 & 406.8 & 356.3 & $3.651 \cdot 10^{6}$ & 3.086 & $1.779 \cdot 10^{-8}$ \\
\hline${ }^{1} S_{0}(p p)$ & 0.499 & 134.7 & 371.7 & $6.763 \cdot 10^{5}$ & 0.421 & $9.564 \cdot 10^{-7}$ \\
\hline
\end{tabular}




\section{Figure captions}

Fig. 1. Phase shifts (solid line) in the ${ }^{3} S_{1}$ chanel for np scattering, compared to the experimental data (points) [32].

Fig.2. Phase shifts (solid line) in the ${ }^{1} S_{0}$ chanel for $n p$ scattering, compared to the experimental data (points) [32].

Fig.3. Phase shifts (solid line) in the ${ }^{1} S_{0}$ chanel for pp scattering, compared to the experimental data (points) [32].

Fig.4. The off-shell behavior of $<\mathbf{p}|T(z)| \mathbf{p}>$ in the ${ }^{3} S_{1}$ chanel for np scattering. The solid curves corresponds to the model with generalized interaction operator (49), compared to the model with Yamaguchi potential with parameters given in [30] (dashed line). 